Neves Fördersystem für erneuerbare Energien in Schweden

\title{
Gegen den Trend
}

\section{In Schweden betrug der Stromanteil der erneuerbaren Energien durch die historisch gewachsenen Nutzung großer Wasserkraft bis Ende 2000 fast sechzig Prozent. Im Ausbau anderer erneverbarer Energien hat sich das Land allerdings bislang schwer getan. Ob das im Frühling eingeführte Quotensystem erfolg- reicher ist als bisherige Förderversuche, ist offen. Sicher ist nur, dass es nicht dem EU-weiten Trend zur Förderung durch Einspeisevergütung entspricht.}

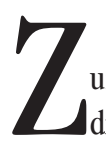
Von Stefan Körner und Danyel Reiche um 1. Mai diesen Jahres hat die schwedische Regierung nach langer Unsicherheit ein Zertifikatshandelssystem für Strom aus erneuerbaren Quellen eingerichtet. Allen Stromverbrauchern - mit Ausnahme energieintensiver Industrien - wurde auferlegt, einen Anteil des von ihnen verbrauchten Stroms, dieses Jahr 7,4 Prozent, aus regenerativer Erzeugung zu beziehen und dies durch den Erwerb von Zertifikaten nachzuweisen. Solche Zertifikate werden an die Erzeuger von regenerativem Strom pro erzeugter Megawattstunde ausgegeben. Zertifikate werden für Strom aus Windkraft, Solarenergie, geothermischer Energie, Biomasse und Wellenkraft vergeben. Strom aus Wasserkraft erhält nur unter bestimmten Bedingungen Zertifikate. Anlagen bis zu einer Leistung von 1,5 Megawatt, neue Wasserkraftwerke, wieder in Betrieb genommene oder erweiterte Anlagen und solche, die ohne Förderung nicht wirtschaftlich arbeiten könnten, werden gefördert.

Die Stromerzeuger können die Zertifikate an die Versorgungsunternehmen und Endkunden weiter verkaufen und erhalten so eine zusätzliche Vergütung für ihren Strom. Der Preis für die Zertifikate wird am Markt gebildet, jedoch wird er nach unten und oben durch einen staatlich garantierten Abnahmepreis und für nicht erfüllte Quotenverpflichtungen zu leistende Strafzahlungen begrenzt. Für nicht verkaufte Zertifikate aus dem Jahr 2003 erhalten die Erzeuger im nächsten Jahr 60 Schwedische Kronen (SEK), ungefähr sechseinhalb Euro. Der garantierte Mindestpreis sinkt jährlich bis 2008 auf Null ab.

\section{- Strafzahlungen vorgesehen}

Die Strafzahlung für fehlende Zertifikate beträgt 150 Prozent des durchschnittlichen Zertifikatspreises des vergangenen Jahres, 2004 aber höch- stens 175 SEK, etwa 19 Euro und 2005 maximal 240 SEK, etwa 26 Euro. Stromerzeuger, die am Zertifikatshandel teilnehmen wollen, müssen sich bei der schwedischen Energiebehörde anmelden und von ihr die Genehmigung zur Teilnahme erhalten. Nur wenn der Endverbraucher nicht ausdrücklich wünscht, sich um die Erfüllung der Quotenverpflichtung selbst zu kümmern, nimmt der Stromversorger seinen Endkunden den Nachweis ab. Dazu erwirbt er die Zertifikate, die der abgegebenen Strommenge entsprechen, legt die Kosten dafür auf die Stromrechnung um und weist diesen Posten gesondert auf der Stromrechnung aus. Endverbraucher, die sich um ihrer Quotenverpflichtung selbst kümmern wollen, müssen sich für eine jährliche Gebühr registrieren lassen.

Die schwedische Regierung plant, die Quotenverpflichtung auf 16,9 Prozent im Jahr 2010 zu steigern. Sie erhofft sich davon die zusätzliche Erzeugung von zehn Tetrawattstunden (TWh) erneuerbarer Energie jährlich. Der momentane Rahmen von 60 bis 175 SEK pro Zertifikat ist, verglichen mit den garantierten Zahlungen des deutschen Erneuerbare-Energien-Gesetzes, eher niedrig und weniger sicher. Die Tatsache, dass verschiedene Erzeugungsarten gleich hoch vergütet werden, wird dazu führen, dass der Löwenanteil der zehn TWh durch den vergleichsweise kostengünstigen Ausbau von Wind- und in Ansätzen Wasserkraft sowie Biomasse erzeugt werden wird.

Bemerkenswert ist der Wechsel zum Quotensystem, da Quotensysteme innerhalb der Europäischen Union als Fördermodelle für erneuerbare Energien nach einer kurzen Hochphase momen$\tan$ wieder weniger Anwendung finden (1). Nur von 1998 bis 2002 führten deutlich mehr Länder Quoten- als Einspeisevergütungsmodelle ein, nachdem Einspeisevergütungen bis zum Jahr 1998 das dominierende Förderinstrument waren.
Der Hauptgrund für das fünfjährige „Hoch“ dürfte in einer institutionalisierten Diffusion durch die Europäische Kommission liegen, die von 1998 an aus ihrer Präferenz für Quotenmodelle keinen Hehl machte, da sie ihres Erachtens wettbewerbsund marktkonformer seien.

Viele Länder gingen davon aus, dass die im Aushandlungsprozess befindliche Richtlinie zur Stromerzeugung aus erneuerbaren Energien eine Vorgabe zur Anwendung von Quotenmodellen enthalten würde. Zudem bestand rechtliche Unsicherheit in bezug auf die Anwendung von Einspeisevergütungen, da das Energieversorgungsunternehmen PreussenElektra 1998 Klage gegen das deutsche Modell der Einspeisevergütung vor dem Europäischen Gerichtshof eingereicht hatte.

\section{EU lässt alle Fördermodelle zu}

Nachdem dieser im März 2001 jedoch entschieden hatte, dass die deutsche Einspeisevergütung keine unerlaubte Subvention sei und die im Oktober 2001 verabschiedete EU-Richtlinie ausdrücklich keine Festlegung auf ein bestimmtes Fördermodell enthielt, ist die Unsicherheit beseitigt worden. Seither haben unter anderem Österreich, Frankreich, Tschechien und Estland Einspeisevergütungen neu eingeführt.

Insofern ist eine neue Hochphase von Einspeisevergütungsmodellen nicht unwahrscheinlich. Unter den EU-Beitrittsstaaten ist Polen das einzige Land mit Quotenmodell, während mit Estland, Tschechien, Slowenien, Slowakei und Ungarn sich bereits fünf Länder für die Einspeisevergütung entschieden haben. In der EU-15 wenden neben Schweden nur Belgien, Italien und Großbritannien ein Quotensystem an. In der EU-27 finden in 16 Ländern in der einen oder anderen Form Mindestpreissysteme Anwendung. Es bleibt abzuwarten, ob sich der schwedische „Sonderweg“ bewährt.

\section{Anmerkung}

(1) Vgl. dazu ausführlicher Reiche, Danyel (Hg.): Handbook of Renewable Energies in the European Union, Frankfurt/ Main 2002 sowie Reiche, Danyel (Hg.): Handbook of Renewable Energies II - Case Studies of all Accession States, Frankfurt 2003.

\section{Die Autoren}

Dr. Danyel Reiche und Stefan Körner sind Mitarbeiter an der Forschungsstelle für Umweltpolitik der FU Berlin. Kontakt: Forschungsstelle für Umweltpolitik, Freie Universität Berlin, Ihnestraße 22, 14195 Berlin, E-Mail: DReiche947@aol.com 
(c) 20I0 Authors; licensee IÖW and oekom verlag. This is an article distributed under the terms of the Creative Commons Attribution Non-Commercial No Derivates License (http://creativecommons.org/licenses/by-nc-nd/3.o/), which permits unrestricted use, distribution, and reproduction in any medium, provided the original work is properly cited. 